\title{
BMJ Open How Australian general practitioners engage in discussions about alcohol with their patients: a cross-sectional study
}

\author{
Emma R Miller, ${ }^{1}$ Imogen J Ramsey, ${ }^{1}$ Ly Thi Tran, ${ }^{1}$ George Tsourtos, ${ }^{1}$ \\ Genevieve Baratiny, ${ }^{2}$ Ramesh Manocha, ${ }^{3}$ Ian N Olver ${ }^{4}$
}

To cite: Miller ER Ramsey IJ, Tran LT, et al. How Australian general practitioners engage in discussions about alcohol with their patients: a crosssectional study. BMJ Open 2016;6:e013921. doi:10.1136/bmjopen-2016013921

- Prepublication history and additional material is available. To view please visit the journal (http://dx.doi.org/ 10.1136/bmjopen-2016013921).

Received 18 August 2016 Revised 24 October 2016 Accepted 27 October 2016

CrossMark

\begin{abstract}
${ }^{1}$ Department of Public Health, Flinders University, Adelaide, South Australia, Australia ${ }^{2}$ University of Southern Queensland, Toowoomba, Queensland, Australia ${ }^{3}$ University of Sydney, Sydney, New South Wales, Australia

${ }^{4}$ Division of Health Sciences, University of South Australia, Adelaide, South Australia, Australia
\end{abstract}

Correspondence to Dr Emma R Miller; emma.miller@flinders.edu.au

\section{ABSTRACT \\ Objective: This study aimed to investigate factors that inhibit and facilitate discussion about alcohol between general practitioners (GPs) and patients.}

Design: Data analysis from a cross-sectional survey. Setting and participants: 894 GP delegates of a national health seminar series held in five capital cities of Australia in 2014.

Main outcome measures: Likelihood of routine alcohol enquiry; self-assessed confidence in assessing and managing alcohol issues in primary healthcare.

Results: Most GPs (87\%) reported that they were likely to routinely ask patients about their alcohol consumption and had sufficient skills to manage alcohol issues (74\%). Potential barriers to enquiring about alcohol included perceptions that patients are not always honest about alcohol intake (84\%) and communication difficulties (44\%). 'I usually ask about alcohol' was ranked by $36 \%$ as the number one presentation likely to prompt alcohol discussion. Altered liver function test results followed by suspected clinical depression were most frequently ranked in the top three presentations. Suspicious or frequent injuries, frequent requests for sickness certificates and long-term unemployment were ranked in the top three presentations by $20 \%$ or less. Confidence in managing alcohol issues independently predicted likelihood to 'routinely ask' about alcohol consumption. Lack of time emerged as the single most important barrier to routinely asking about alcohol. Lack of time was predicted by perceptions of competing health issues in patients, fear of eliciting negative responses and lower confidence in ability to manage alcoholrelated issues.

Conclusions: Improving GPs' confidence and ability to identify, assess and manage at-risk drinking through relevant education may facilitate greater uptake of alcohol-related enquiries in general practice settings. Routine establishment of brief alcohol assessments might improve confidence in managing alcohol issues, reduce the time burden in risk assessment, decrease potential stigma associated with raising alcohol issues and reduce the potential for negative responses from patients.

\section{Strengths and limitations of this study}

- This study is one of only a few to have examined factors facilitating and inhibiting general practitioners (GPs) routinely asking about their patients' alcohol consumption in Australian settings.

- Study limitations include the following: selfselected sample, target population likely to have enhanced awareness of the preventive practice, two approaches to data collection and limitations of number of variables collected.

- However, our analytical approach identified important predictors of alcohol prevention and our findings are likely to be relevant to other countries in which preventive healthcare is primarily provided by GPS.

\section{INTRODUCTION}

In Australia, alcohol consumption accounts for $3 \%$ of the total burden of disease and injury. ${ }^{1}$ Hazardous alcohol consumption has been causally liked to more than 60 different health conditions and contributes to 3450 deaths and 85435 disability-adjusted life years (DALYs) per year in Australia. ${ }^{2}$ The high population prevalence of cofactors, such as cardiovascular disease, greatly escalate alcohol-associated complications and mean that is likely that the true burden of chronic harmful and hazardous drinking will never be accurately estimated. ${ }^{3}$ Approximately one-quarter of Australian adult general practice attendees engage in at-risk drinking, defined as drinking behaviours that place an individual at increased risk of alcohol-related harm, ${ }^{4}$ yet less than one in three Australian women and one in six Australian men with documented alcohol dependence actively seek treatment. ${ }^{56}$

Primary care is a key setting for the reduction of alcohol-related harm, with general 
practitioners (GPs) ideally positioned to prevent, detect and manage at-risk drinking. The Royal Australian College of General Practitioners Guidelines for preventative activities in general practice (the Red Book) recommend that 'All patients should be asked about the quantity and frequency of alcohol intake from age 15 years' ( p. 75). ${ }^{6}$ However, underdetection of harmful drinking in general practice has been reported ${ }^{78}$ and research suggests that few GPs have embedded early detection practices into routine care..$^{9} 10$

Common barriers to identifying and managing at-risk drinking include the following: brief consultations, stigma attached to diagnosis, patient sex (women are less likely to receive treatment) and GPs' perceived lack of skills. ${ }^{1-14}$ Little is known about how GPs initiate discussions about alcohol with their patients in Australian general practice settings; ${ }^{15}$ however, Tam et al $\bar{l}^{7}$ suggest that GPs' willingness to ask about alcohol use may be influenced by social and cultural attitudes regarding alcohol consumption, and concerns about the consultation dynamics between patient and doctor. Identifying potentially harmful alcohol consumption is a necessary first step in managing those issues once they are detected and there is some evidence to suggest that the two concepts, detection and management, may be linked via reduced GP confidence in their ability to undertake follow-up interventions. ${ }^{16}$ In a review of the worldwide literature, Yoast $e t a l^{17}$ found that lack of selfefficacy was the chief reason proposed by physicians for not providing care around substance use. They state ( $p$. 83), 'They do not have familiarity or experience with screening and intervention techniques, lack confidence in their skills to intervene, and doubt the effectiveness of the help they had provided to patients'. In their survey of more than 2300 GPs in eight European countries, Anderson et $a l^{18}$ found that GPs with higher education about alcohol and GPs with confidence in managing patients with alcohol issues were more likely to manage such patients, while those who believed alcohol was a 'disease' or those who viewed drinking as a personal, rather than medical, responsibility tended to manage fewer patients with alcohol issues.

According to Knox et $a l^{19}$ almost $90 \%$ of Australians visit a GP on at least one occasion each year with an average of six visits per person annually, providing GPs with substantial opportunity to discuss alcohol behaviour with their patients. Given their substantiated efficacy in prevention, more information is required from GPs regarding their perceptions around discussing alcohol consumption. This may inform the development of strategies assisting GPs to discuss alcohol intake with their patients more frequently, effectively manage any alcohol issues identified and ultimately reduce the impact of alcohol-related harm in the population.

We aimed to identify factors facilitating and inhibiting routine alcohol enquiry among Australian GPs by exploring their current practice and perceptions around alcohol assessment and management. The proportion of
GPs reporting sufficient skills and the ability and confidence to identify, assess and manage their patients' alcohol consumption were examined. Barriers to routine alcohol enquiry and typical presentations prompting alcohol-related discussions were also investigated.

\section{METHODS}

A questionnaire was administered in paper-based and online format, the latter was developed using Qualtrics survey software. The items were informed by the literature and developed in consultation with a reference group comprised of Australian experts in the field of alcohol and general practice. The 12-member reference group (additional to the research team) included members from the Royal Australian College of General Practitioners, key researchers in the field of alcohol from universities around Australia and representatives from key organisations involved in research and education on alcohol and drugs nationally. The survey was piloted among the research team and its networks, which included affiliated GPs, and subsequently refined for usability.

\section{The survey}

To reduce the burden on participants, the survey was presented on one page containing four types of questions. The first part of the survey included demographic information such as sex, age, years worked in general practice and employment fraction (full time or parttime). Forming the two main outcome variables, participants were asked to indicate how likely they were, in a usual month of general practice, to routinely ask about their patients' alcohol consumption, as well as their confidence and ability to assess and manage at-risk drinking. In the third grouping of questions, participants rated their level of agreement with 14 statements regarding potential barriers and facilitators to asking about alcohol on a 5-point Likert Scale (1='strongly disagree' to $5=$ 'strongly agree'). Participants also ranked the top three patient presentations that would prompt them to ask about a patient's alcohol consumption, from 12 possible options (an 'other' option was also offered). Other questions relating to cancer and alcohol will form part of a separate report (see the survey in online supplementary file).

\section{Participants}

Registered Australian GPs who attended a national series of seminars on the topic of women's and children's health in Adelaide, Brisbane, Melbourne, Sydney or Perth (five capital cities) in 2014 were eligible to participate in the study. As representativeness cannot be assumed in a self-selected sample, we aimed to recruit a sufficiently large sample to allow for meaningful stratified statistical analysis, according to key demographic questions that were included in the questionnaire. Paper-based questionnaires were provided to seminar 
attendees in Perth, Brisbane and Adelaide to complete in their own time during the seminar. The conference organiser entered de-identified data from the individual paper questionnaires into an encrypted spreadsheet for de-identified analysis. GPs who attended the seminars in Sydney and Melbourne, held earlier in 2014, received an email invitation to complete the questionnaire online. Examination of demographic information against repeated internet protocol (IP) addresses indicated that there were no duplicate entries in the online data set.

\section{Data analysis}

A descriptive analysis was conducted to examine measures of central tendency, range and type of distribution for continuous variables, and percentage distribution for categorical variables. The online and paper-based groups were compared in terms of demography to determine whether they could be combined for analysis. To compensate for small counts in the extreme categories of the independent variables (and many of the independent variables), Likert Scale agreement categories were dichotomised to create measures of agreement in subsequent analyses-combining 'strongly agree' with 'agree' and combining 'neither', 'disagree' and 'strongly disagree'. Likert Scales for likelihood ('very likely' to 'very unlikely') were dichotomised in a similar way.

We developed agreement ratios with CIs using univariate and multivariate techniques where appropriate. $\chi^{2}$ tests were conducted to explore the bivariate associations between the 14 statements forming potential barriers and facilitators and the main outcome variables (likelihood to routinely enquire about alcohol and confidence in ability to assess and manage at-risk drinking). Binary logistic regression was performed to identify factors independently predicting the two outcomes. To control for potential bias and confounding associated with their over-representation in the sample, sex and duration of general practice were included in all multivariate models. All data were analysed at the significance level of 0.05 . As the responses were relatively complete, analyses excluded missing data. Data were analysed using
Stata (release V.13, Stata Corporation, College Station, Texas, USA).

\section{RESULTS}

Completed questionnaires were returned by 894 of the $\sim 2570$ eligible GPs (35\% response rate). Of these, 554 (62\%) completed paper-based questionnaires and 340 $(38 \%)$ completed the questionnaire online. Female GPs were over-represented in the sample, with a male GP to female GP ratio of 1:4 (table 1). The mean participant age was 48 years and the mean general practice experience was 17 years. Over half the sample (53\%) had worked mostly part-time during their careers, 29\% mostly full time and $18 \%$ equally full time and part-time. Overall, female GPs were significantly younger than male GPs in this group-mean 46.5 vs 52.2 years-d t (857) $-5.64, \mathrm{p}<0.001$ (data not shown).

The online and paper-based groups showed similar distributions of sex and employment fraction. Among male GPs, the two groups did not differ by age or years of experience. The differences between the two groups were among female participants only. The mean age of female GPs was 3 years higher in the online group, which corresponded with three more years of general practice experience. Although statistically significant, the 3-year age differential and associated difference in duration of practice is unlikely to be meaningful. Given that sex and duration of practice would be controlled for in the analyses, data from the two groups were combined.

The vast majority of GPs reported that they were likely to routinely ask patients about alcohol consumption in a usual month, and had sufficient skills and confidence to assess and manage at-risk drinking (table 2). Participants were far less likely to agree they were able to 'tell' if their patients had alcohol issues; particularly female participants. Female GPs were also were less likely to report being confident in their ability to assess and manage at-risk drinking in their patients.

In terms of potential barriers and facilitators to discussing alcohol with patients, $84 \%$ of participants agreed

Table 1 Characteristics of general practitioner participants by method of data collection

\begin{tabular}{|c|c|c|c|c|}
\hline & $\begin{array}{l}\text { All participants } \\
\mathrm{N}=894\end{array}$ & $\begin{array}{l}\text { Paper-based } \\
N=554\end{array}$ & $\begin{array}{l}\text { Online survey } \\
\mathrm{N}=340\end{array}$ & p Value* \\
\hline Females-n (\%) & $725(81)$ & $445(82)$ & $270(79)$ & 0.314 \\
\hline Age in years-mean (SD) & $47.6(11.4)$ & $46.4(10.8)$ & $49.4(12.1)$ & $<0.001$ \\
\hline Females & 46.5 (10.9) & $45.2(10.5)$ & 48.5 (11.2) & $<0.001$ \\
\hline Males & $52.2(12.3)$ & $51.8(10.4)$ & $52.4(14.7)$ & 0.788 \\
\hline General practice experience in years-mean (SD) & $17.2(12.2)$ & $16.2(11.5)$ & $18.8(13.1)$ & $<0.001$ \\
\hline \multicolumn{5}{|l|}{ Pattern of work-n (\%) } \\
\hline Almost/mostly full-time & $254(29)$ & $165(30)$ & $89(28)$ & 0.489 \\
\hline Almost/mostly part-time & $465(53)$ & $286(52)$ & $179(56)$ & \\
\hline Half and half & 153 (18) & 101 (18) & 52 (16) & \\
\hline
\end{tabular}


Table 2 General practitioners' self-evaluation of their practice, skills, ability and confidence $(\mathrm{N}=894)$

\begin{tabular}{|c|c|c|c|c|}
\hline & $\begin{array}{l}\text { All GPs } \\
\%\end{array}$ & $\begin{array}{l}\text { Males } \\
\%\end{array}$ & $\begin{array}{l}\text { Females } \\
\%\end{array}$ & p Value* \\
\hline $\begin{array}{l}\text { Likely/very likely to routinely ask about alcohol consumption over a usual } \\
\text { month (missing } n=24,2.7 \% \text { ) }\end{array}$ & 87.4 & 85.4 & 87.8 & 0.395 \\
\hline $\begin{array}{l}\text { Agree/strongly agree have sufficient skills to assess at-risk drinking } \\
\text { (missing } n=51,5.7 \%)\end{array}$ & 73.7 & 75.9 & 73.1 & 0.467 \\
\hline $\begin{array}{l}\text { Agree/strongly agree able to tell if patients have alcohol issues (missing } \\
n=58,6.5 \% \text { ) }\end{array}$ & 25.5 & 37.3 & 22.7 & $<0.001$ \\
\hline $\begin{array}{l}\text { Confident/very confident in assessing and managing at-risk drinking } \\
\text { (missing } n=72,8.0 \%)\end{array}$ & 53.9 & 66.2 & 51.0 & 0.001 \\
\hline
\end{tabular}

with the statement that patients are not always truthful about their alcohol consumption, and $44 \%$ agreed that communication difficulties prevented alcohol discussion (figure 1). Other statements where greater than one-third of GPs agreed were the presence of competing health issues and that patients do not want to hear about health risks associated with drinking. Approximately $30 \%$ of participants agreed that they 'raise alcohol issues with patients depending on the patient's age, sex or ethnicity' and they were 'more likely to bring up alcohol issues with patients from particular occupations or from particular socioeconomic groups'. Fewer than half of male and female GPs agreed they were sufficiently informed about alcohol misuse and related issues during their medical education. Female GPs were more likely than male GPs to agree that patients do not always tell the truth about alcohol $(86 \%$ vs $\left.78 \% ; \chi^{2}=6.107 ; \mathrm{p}=0.013\right)$, and communication barriers ( $40 \%$ vs $\left.31 \% ; \chi^{2}=7.306 ; \mathrm{p}=0.007\right)$ and competing health issues $\left(47 \%\right.$ vs $\left.35 \% ; \chi^{2}=4.852 ; p=0.028\right)$ can prevent alcohol discussion. A higher proportion of male GPs agreed that they were 'usually able to tell' if a patient had alcohol issues $\left(37 \%\right.$ vs $\left.23 \% ; \chi^{2}=14.595 ; \mathrm{p}<0.001\right)$ and that they raised alcohol issues depending on the patients' age, sex or ethnicity $\left(39 \%\right.$ vs $30 \% ; \chi^{2}=4.583$; $\mathrm{p}=0.032)$. Overall, $37 \%$ of the participants agreed that regular alcohol, even at light levels, can lead to cancer but female GPs were more likely to agree than male GPs ( $40 \%$ vs $36 \% ; \chi^{2}=9.199 ; p=0.002$ ).

When ranking the top three presentations that would prompt an alcohol-related enquiry (figure 2), the two options most frequently ranked by participants at number one were 'I usually ask about alcohol consumption' $(36 \%)$ and 'patient smells of alcohol' $(30 \%)$. The

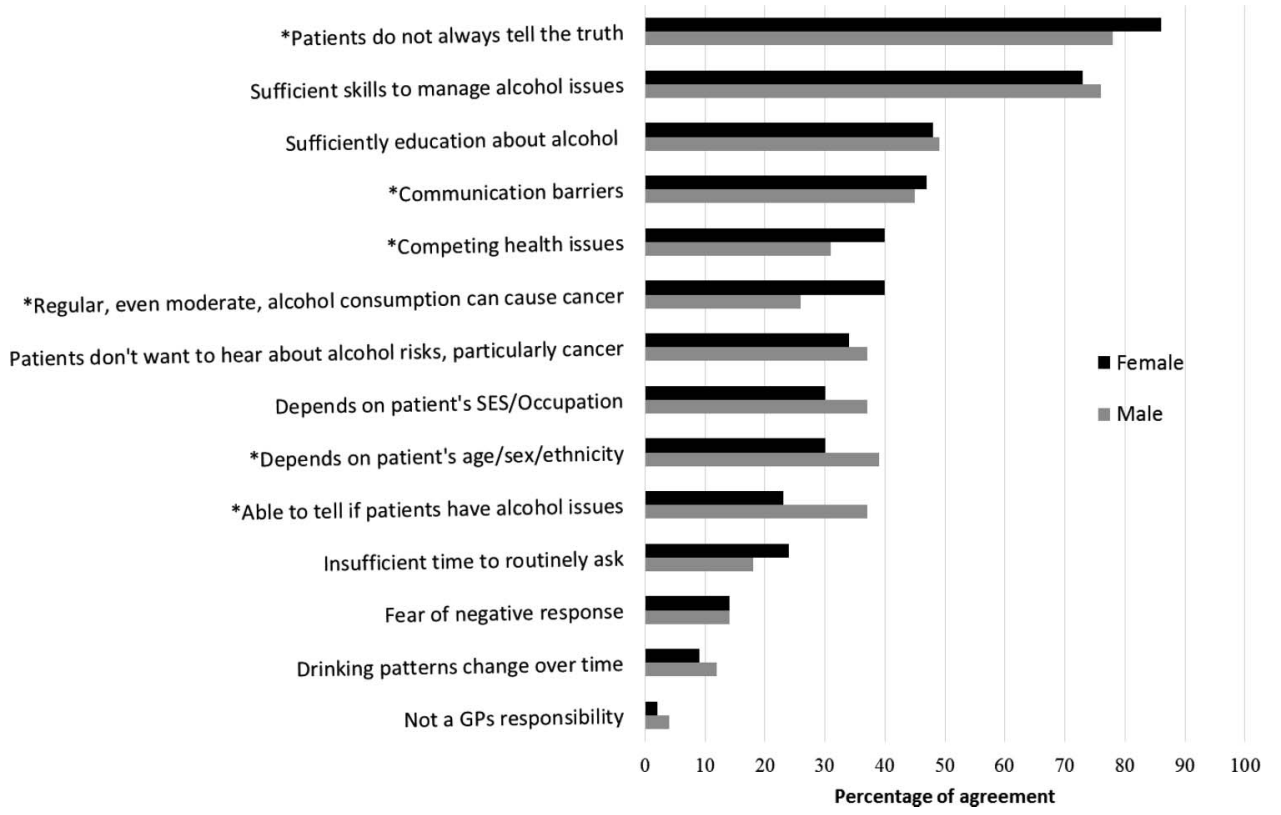

Figure 1 General practitioner (GP) agreement with statements related to discussing alcohol consumption with patients. *Significant 2-tailed difference- $-p<0.05$. 


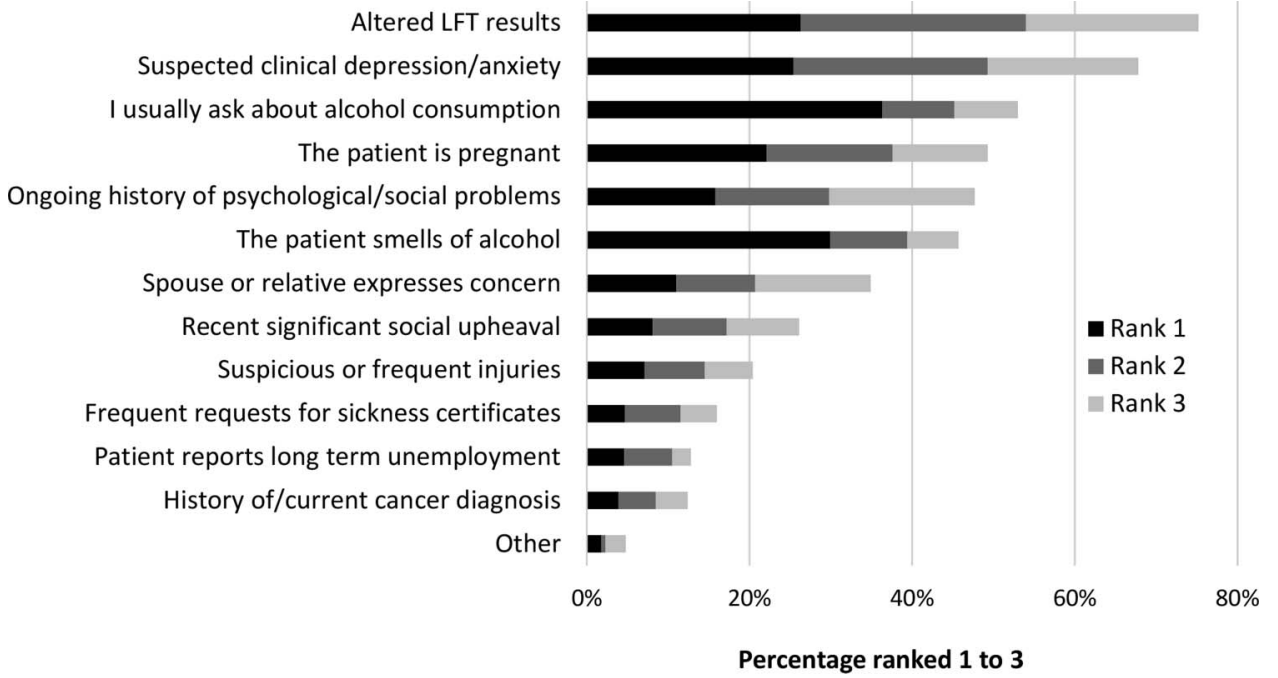

Figure 2 Presentations most likely to prompt alcohol discussions-general practitioner rankings 1-3. LFT, liver function test.

most common second and third rankings were 'altered liver function test (LFT) results' followed by 'suspected clinical depression/anxiety'. These presentations received the most top three rankings $(75 \%$ and $68 \%$, respectively), ahead of 'I usually ask about alcohol consumption' $(53 \%)$. Presentations ranked in top three by the fewest number of participants were 'suspicious or frequent injuries' (20\%), 'frequent requests for sickness certificates' (16\%), 'patient reports long-term unemployment' (13\%) and 'history of/current cancer diagnosis' $(12 \%)$.

\section{Bivariate analyses}

Factors bivariately associated with GPs reporting they were likely to 'routinely ask about alcohol consumption' are outlined in table 3. Self-reported confidence in assessing and managing at-risk drinking, agreement they had sufficient education and sufficient skills to assess at-risk drinking were most strongly positively associated with 'routinely ask about alcohol'. Lack of time, competing patient health issues and fear of negative responses were most strongly negatively associated with likelihood to 'routinely ask about alcohol'.

Table 3 Factors bivariately associated with routine alcohol enquiry, confidence and skills ( $N=799)$

\begin{tabular}{|c|c|c|c|}
\hline Outcome & Factor & Agreement ratio $(95 \% \mathrm{Cl})$ & p Value* \\
\hline \multicolumn{4}{|c|}{ Likely to routinely ask about alcohol: } \\
\hline & Confidence to assess/manage & $1.17(1.11$ to 1.24$)$ & $<0.001$ \\
\hline & Able to tell if patients have problems & $1.10(1.05$ to 1.16$)$ & 0.001 \\
\hline & Sufficient skills to assess & 1.09 (1.02 to 1.17$)$ & 0.007 \\
\hline & Sufficient education & 1.08 (1.03 to 1.14$)$ & 0.004 \\
\hline & Agrees regular alcohol a cancer risk & 1.07 (1.01 to 1.13$)$ & 0.007 \\
\hline & Communication issues a barrier & 1.06 (1.01 to 1.12$)$ & 0.025 \\
\hline & Ask depending on occupation or SES & 0.92 (0.86 to 0.98$)$ & 0.003 \\
\hline & Fear of negative response & $0.91(0.83$ to 1.00$)$ & 0.027 \\
\hline & Competing health issues & 0.86 (0.81 to 0.92$)$ & $<0.001$ \\
\hline & Lack of time & $0.77(0.70$ to 0.85$)$ & $<0.001$ \\
\hline \multicolumn{4}{|c|}{ Confidence in ability to assess/manage: } \\
\hline & Sufficient skills to assess & $2.17(1.75$ to 2.70$)$ & $<0.001$ \\
\hline & Able to tell if patients have problems & 1.50 (1.33 to 1.69$)$ & $<0.001$ \\
\hline & Sufficient education & 1.48 (1.30 to 1.69$)$ & $<0.001$ \\
\hline & Male sex & $1.30(1.14$ to 1.49$)$ & $<0.001$ \\
\hline & Patients do not always tell the truth & $0.84(0.72$ to 0.98$)$ & 0.036 \\
\hline & Patients do not want to hear & $0.83(0.72$ to 0.96$)$ & 0.008 \\
\hline & Competing health issues & $0.73(0.63$ to 0.84$)$ & $<0.001$ \\
\hline & Fear of negative response & $0.72(0.58$ to 0.91$)$ & 0.002 \\
\hline & Lack of time & $0.68(0.57$ to 0.83$)$ & $<0.001$ \\
\hline
\end{tabular}


Strongly associated with 'routinely asking about alcohol', self-reported confidence in their ability to assess and manage alcohol issues in their patients also formed an exploratory outcome for bivariate analysis. Agreement they had sufficient education and sufficient skills to assess at-risk drinking, able to identify patients with alcohol issues and being male GP were most positively associated with GPs agreeing they were confident to assess and manage at-risk drinking. Agreement that there was a lack of time for alcohol discussions, fear of negative responses to raising the issue and competing patient health issues were most negatively associated with confidence in managing at-risk drinking.

\section{Multivariate analyses}

We built $\log$ binomial models to identify independent predictors of the main outcomes 'routinely asking about alcohol consumption' and 'confidence in ability to assess/manage alcohol issues'. All models included sex and years of experience to control for the overrepresentation of female GPs in the study, and the longer practice duration of female GPs in the online group. Factors bivariately associated with GPs likelihood to ask about alcohol consumption in the bivariate analysis were classified as either potential facilitators (eg, agreement with statements related to confidence and sufficient education, skills and ability) or potential barriers (eg, agreement with statements related to lack of time, competing health issues, fear of negative responses, etc). Separate binomial models were built for each category of predictors and outcomes, routinely asking about alcohol and confidence in assessing and managing alcohol issues.

For the outcome 'routinely asking about alcohol', the following factors were no longer significant, after adjusting for other factors, and fell out of all the models: agreeing they had sufficient skills; agreeing they were sufficiently educated to manage alcohol issues; asking depending on the patients' age, sex and ethnicity; fear of negative responses from patients; and anticipating communication difficulties. In our final model (table 4), confidence in their ability to manage and assess alcohol was the most important factor (agreement ratio of 1.12, $10 \%$ absolute difference). Agreeing they were 'usually able to tell' if patients have alcohol issues, and being female GP also independently predicted likelihood to routinely ask about alcohol consumption. Lack of time was a significant barrier to routinely asking, with those agreeing with the statement about lack of time being $20 \%$ less likely to 'routinely ask' (agreement ratio 0.80 ), with an absolute agreement difference of $18 \%$.

We followed the same procedure in modelling on the outcome 'confidence in assessing and managing alcohol issues'. Being male GP, sufficient education, sufficient skills and usually being able to identify alcohol issues in patients remained significant predictors of confidence to assess and manage alcohol issues. Agreeing that patients do not always tell the truth about alcohol and that lack of time prevents alcohol discussions were significant predictors of not reporting confidence in assessing and managing alcohol issues. After 'patients do not always tell the truth about alcohol' fell out, our final

Table 4 Models predicting general practitioners' likelihood to routinely ask about alcohol use, confidence to assess and manage alcohol issues and lack of time to ask about alcohol $(\mathrm{N}=805)$

\begin{tabular}{|c|c|c|c|}
\hline Model* $^{*}$ & $\begin{array}{l}\text { Adjusted agreement } \\
\text { ratio }(95 \% \mathrm{Cl})\end{array}$ & $\begin{array}{l}\text { Adjusted agreement } \\
\text { difference }(95 \% \mathrm{Cl})\end{array}$ & p Value† \\
\hline \multicolumn{4}{|l|}{ Likely to routinely ask about alcohol consumption: } \\
\hline Confidence in ability to assess and manage alcohol issues & $1.12(1.07$ to 1.18$)$ & $0.10(0.05$ to 0.14$)$ & $<0.001$ \\
\hline Can usually tell if patients have alcohol issues & $1.11(1.08$ to 1.14$)$ & $0.08(0.05$ to 0.10$)$ & $<0.001$ \\
\hline Female sex & 1.09 (1.04 to 1.15$)$ & 0.07 (0.02 to 0.11$)$ & $<0.001$ \\
\hline Not enough time to ask & $0.80(0.73$ to 0.88$)$ & $-0.18(-0.25$ to -0.11$)$ & $<0.001$ \\
\hline \multicolumn{4}{|l|}{ Confidence in ability to assess and manage alcohol issues: } \\
\hline Has sufficient skills to assess alcohol problems & 1.89 (1.52 to 2.34$)$ & $0.28(0.21$ to 0.36$)$ & $<0.001$ \\
\hline $\begin{array}{l}\text { Was sufficiently informed about alcohol during medical } \\
\text { education }\end{array}$ & $1.27(1.13$ to 1.44$)$ & $0.14(0.07$ to 0.20$)$ & $<0.001$ \\
\hline Can usually tell if patients have alcohol issues & $1.26(1.13$ to 1.41$)$ & $0.16(0.09$ to 0.24$)$ & $<0.001$ \\
\hline Male sex & $1.15(1.03$ to 1.28$)$ & $0.11(0.03$ to 0.18$)$ & 0.010 \\
\hline Not enough time to ask & $0.78(0.65$ to 0.93$)$ & $-0.17(-0.24$ to -0.10$)$ & 0.005 \\
\hline \multicolumn{4}{|c|}{ Usually not enough time to enquire about each patient's alcohol intake: } \\
\hline $\begin{array}{l}\text { Not always discussed because not a priority in patients with } \\
\text { competing health issues }\end{array}$ & $1.91(1.46$ to 2.50$)$ & 0.15 (0.09 to 0.22$)$ & $<0.001$ \\
\hline $\begin{array}{l}\text { Does not always raise alcohol consumption because it can } \\
\text { elicit negative responses }\end{array}$ & $1.56(1.18$ to 2.06$)$ & $0.14(0.04$ to 0.24$)$ & 0.002 \\
\hline Confidence in ability to assess and manage alcohol issues & $0.65(0.50$ to 0.85$)$ & $-0.10(-0.15$ to -0.04$)$ & 0.001 \\
\hline
\end{tabular}

${ }^{*}$ All factors adjusted for sex and practice duration as well as all other factors presented in each model.

†Log binomial models used. 
combined model (table 4) identified that agreeing they had sufficient skills was the strongest predictor of selfreported confidence in assessing and managing alcohol issues (agreement ratio of 1.89; $28 \%$ absolute difference). Agreeing that they had sufficient information about alcohol issues during medical education and that they could usually tell if their patients had alcohol issues, and being male GP were also independent predictors of confidence in assessing and managing alcohol issues. Agreeing that there was not sufficient time to ask about alcohol was a negative predictor of self-reported confidence in managing alcohol issues (agreement ratio of 0.78 ; agreement difference of $17 \%$ ).

Given its continued significance in the above models, the concept of 'lack of time' was further analysed as an outcome factor. Agreeing they had 'sufficient skills' and 'sufficient information', that patients do not always want to hear about alcohol health risks and communication difficulties fell out of the models. In the final model (table 4), once adjusted for sex and duration for practice, competing health issues in patients and fear of negative responses in patients were significant predictors of agreeing there was not always enough time to ask about alcohol. Confidence in ability to assess and manage alcohol issues was negatively associated with agreeing that there was not enough time to discuss alcohol.

\section{DISCUSSION}

Our study is one of only a few to have examined factors facilitating and inhibiting GPs routinely asking about their patients' alcohol consumption in Australian settings. Our findings are likely to be relevant to other countries wherever preventive healthcare is primarily provided by GPs in community settings. Confidence in managing alcohol issues (including being confident in being able to identify alcohol issues) predicted likelihood to 'routinely ask' about alcohol consumption, while lack of time emerged as the single most important barrier. Agreeing that a lack of time prevented alcohol discussions was predicted by prioritisation of health issues and fear of negative responses from patients elicited by raising alcohol issues. Those who reported confidence in their ability to assess and manage alcohol issues were less likely to agree that there was insufficient time.

In contrast to the reported perspectives of GPs elsewhere, ${ }^{20}$ our GP participants overwhelmingly supported the statement that, in a usual month, they were likely to routinely ask patients about their alcohol status. Previous research has noted discrepancies between the perceptions of GPs and those of patients with regard to the frequency of alcohol-related enquiries. According to Litt, ${ }^{21}$ patients' perceptions about how often they are asked about their alcohol intake may be lower than numbers provided by GPs, who tend to overestimate their practice in prevention. In our study, it is possible that 'routinely ask' was not interpreted to mean that GPs universally asked patients at each visit but that they routinely did so in certain circumstances. Indeed, when asked to identify patient presentations that would prompt them to discuss alcohol, only 32\% ranked 'I usually ask about alcohol consumption' within their top three presentations. The presentation rankings (see figure 2) support evidence that GPs tend to rely more on clinical judgements and medical conditions at consultation when deciding to initiate conversations about alcohol with patients, rather than enquiring as routine practice..$^{22}$ Given that from 2013 to 2014, LFTs were only ordered at $2.4 \%$ of GP encounters, and anxiety and depression represented $7 \%$ of problems managed at general practices, ${ }^{4}$ it is likely that a large number of patients with problematic alcohol behaviours could remain undetected should these presentations be the primary prompt for enquiry. Mitchell et $a l^{23}$ concluded that healthcare professionals struggle to identify problem drinking in clinical practice, using clinical judgements to identify half of those with alcohol use disorder based on clinical judgement and accurately noting alcohol use disorder in only a third of actual cases. It is important to note that our participants were asked to rank the top three most important presentations, rather than rate an exhaustive list of potential presentations, and non-selection of an option should not be interpreted as meaning GPs ignored that presentation. Further, among the broad range of presenting problems in general practice, presentations most likely to prompt alcohol discussions are likely to be highly contextual. Yet, finding ways to enhance clinical decision-making in regard to problematic alcohol use is likely to be beneficial given our results.

The proportion of GPs agreeing they had sufficient skills and confidence to assess and manage alcohol issues was higher in our sample than other Australian studies, ${ }^{9}{ }^{24}$ as was the proportion who reported being likely to routinely enquire about patients' alcohol consumption. ${ }^{25}$ Most of our participant GPs indicated confidence in their ability to assess and manage alcohol issues and agreed that this was part of their role in preventive practice. To a separate statement, however, only one in three agreed they were usually able to tell if patients were at risk for problematic alcohol use. A recent study estimated low sensitivity in the detection of harmful alcohol consumption in general practice settings, where only a minority of patients who reported harmful drinking were identified by their $\mathrm{GP}^{26}$ It is possible that GPs interpreted the statement about being able to tell if their patients had alcohol issues differently - for example, either they are able to intuitively know (or guess), regardless of any information offered by the patient, or that they have confidence in their clinical ability to identify such patients. Indeed, agreeing they were 'usually able to tell' if their patients had alcohol issues independently predicted GPs' confidence in their ability to manage alcohol issues and both of these factors independently predicted likelihood to routinely ask about alcohol consumption. Nonetheless, strategies 
aimed at enhancing assessment abilities might increase likelihood of routinely asking about alcohol.

Fewer than half of male and female GPs agreed they were sufficiently informed about alcohol misuse and related issues during their medical education. Agreement to this statement predicted confidence in assessing and managing alcohol issues, which in turn predicted likelihood of GPs routinely enquiring about alcohol. Our findings support previous work highlighting the need for more comprehensive alcohol education $^{27} 28$ and reporting that specialised training can increase the self-efficacy of GPs and promote greater uptake of alcohol assessment. ${ }^{29} 30$

Our results suggest that most GPs believe they are likely to ask about their patients' alcohol consumption; however, these discussions are frequently prompted by clinical circumstances and are therefore yet to be embedded within routine practice (ie, at each new presentation). Rather than ranking the top three presentations that prompt alcohol discussions, it is possible that some GPs may have ranked presentations that can be directly attributed to alcohol. The positioning of 'I usually ask about alcohol consumption' as the first option, however, was likely to have minimised the possibility of misunderstanding. Given the lower proportion of our participants who agreed they are able to tell if their patients had alcohol issues, it is possible that GPs and their patients would benefit from a consistent approach to identifying and assessing patients at risk, informed by research on the challenges to detecting problematic drinking in general practice. ${ }^{26}$ The WHO has developed, and extensively tested, a screening instrument for detecting harmful and hazardous drinking and linked brief intervention for primary healthcare (PHC) providers. ${ }^{31-34}$ The 10-item Alcohol Use Disorder Identification Test (AUDIT) has been well validated in six countries, including Australia, and is linked to brief PHC-based interventions ranging from simple advice about alcohol to brief counselling or referral to specialists for evaluation and treatment. ${ }^{35}$ Despite considerable evidence for the efficacy and cost-effectiveness of PHC-based screening and linked interventions in alcohol use, ${ }^{30}$ 36-40 widespread incorporation of the approach in routine care has not occurred in Australia or elsewhere. ${ }^{30}{ }^{41}$ Similar to our findings, 'lack of time' was identified as a major barrier by GP participants in a Victorian (Australia) evaluation. ${ }^{30}$ Bush et al ${ }^{42}$ subsequently developed a modification of the AUDIT instrument, the 3-item AUDIT-C, which provides a score out of 12 points to reliably identify PHC patients who drink at hazardous levels or who have active alcohol use disorders. The AUDIT-C has been found to perform well in general population surveys and among young people of both sexes ${ }^{434}$ and may provide a rapid first screen, with only higher scores indicating the need for the full AUDIT and linked intervention process. Routine primary screening with the shorter AUDIT-C might be seen as less time-consuming and potentially less intrusive than the full AUDIT has been perceived to be. ${ }^{16}$ In their qualitative study, Tam $e t a l^{7}$ found that GP responses to even the shorter version were largely negative. Future larger studies across a range of PHC settings may find ways to increase the acceptability and usefulness of such tools for routine practice as well as assess their impact on GP confidence in identifying at-risk patients. There is also evidence that the screening process itself may be the more effective component, with positive outcomes not varying according to intensity of the intervention in Emergency Department patients. ${ }^{46}$ Assessing the usefulness of using various formats of brief screening in PHC (eg, whether completed by the patient, GP or practice nurse) for the purpose of facilitating discussion could form the basis for future study.

Brief alcohol screening tools may assist GPs to initiate discussions about alcohol in a non-personalised way. They may also help to address perceptions of lack of time', which emerged as an important barrier to routinely enquiring about alcohol consumption. Given 'lack of time' predicted reduced likelihood to routinely ask and lower confidence in assessing and managing alcohol problems, this concept was further examined and a more complex make-up of the notion of 'lack of time' was suggested. We found that competing health priorities in patients, fear of negative responses elicited from patients in response to raising alcohol issues and lower confidence in alcohol management ability all independently predicted agreement that there was not always sufficient time to discuss alcohol. The time pressure on GPs is real ${ }^{47}$ and, given the number of presenting health issues per patient, it is not surprising that some prioritising would occur. Fear of eliciting negative responses was found to independently predict agreement that there was a lack of time to discuss. Previous qualitative evidence identified fear of upsetting patients and potential threat to the patient-doctor relationship as barriers to discussions of alcohol consumption in primary care. ${ }^{7} 48$ On the whole, patients are reported to be supportive of GPs enquiring about alcohol use, especially those with chronic conditions such as diabetes, oesophageal disease, hypertension and depression. ${ }^{4-51}$ Other evidence, however, validates the apparent apprehension in our participants. Tam et $a \tilde{l}^{2}$ found that willingness to accept GPs raising alcohol issues was highly contextual and could be seen as judgemental and stigmatising. They caution (p. 837), 'We need to be respectful of the beliefs and attitudes that patients and GPs have towards their relationship, and recognise the morally charged nature of alcohol discussions'. Instituting brief alcohol assessment as routine practice may ultimately reduce the potential for negative responses, particularly where GPs link their enquiry to the presenting health issue. ${ }^{52}$

\section{Limitations and recommendations}

Our sample included GPs attending an educational seminar series, which may suggest enhanced awareness of the importance of preventive practice in our 
participants. There was also a disproportionate representation of female GPs in our study, which was due to their over-representation in education series on women and children's health, combined with a tendency for greater survey response from females relative to males in general. ${ }^{53}$ Although this impacts on the representativeness of the overall sample, we recruited a sufficiently large number of GPs (nearly 900) from across Australia to allow for stratified bivariate analyses and we adjusted for sex in the multivariate analyses. Similarities in overall patterns of response in our stratified analyses (see figure 1) provide greater confidence in the overall analytical approach. Unavoidable administrative delays resulted in two approaches being used for data collection-online and paper-based. Although consistency in data collection would have been preferable, comparison of the demographic characteristics of each group provided good evidence of the appropriateness of combining the data for analysis.

Our survey was limited to one page to ensure ease of completion. Although it was informed by the literature, and developed in consultation with experts in the field, the survey did not include previously validated measures. Feedback from the pilot and responses to the survey suggested consistency between the intended meaning of questions and their comprehension; however, confirming our conclusions with further study is recommended. Also due to the brevity of the survey, there were issues not explored that might have allowed greater insight into GP interpretations of concepts such as 'routinely asking about alcohol'. Future studies might consider GPs' drinking behaviours and explore the relationship between their own attitudes and how they initiate alcohol-related discussions with patients. Investigating GPs' interpretations and understanding of the Red Book guidelines, and whether they currently use (or might consider using) brief assessment tools such as the AUDIT-C might also provide insight into their practice in this area. Further understanding of GP knowledge of, and access to, specialist alcohol services would also be valuable. Further work focusing on the contribution of factors external to the GP, such as patient characteristics and expectations, would also allow for a more complete picture of this issue.

Our analyses identified independent predictors of likelihood to routinely ask about alcohol and confidence in managing alcohol issues; however, the absolute differences were not large-possibly indicating that not all contributing factors were uncovered. Importantly, however, some of the contributory factors identified in our study are potentially modifiable. More research on this complex issue is required; however, our findings could help to inform the development of actions aimed at enhancing preventive practice.

\section{CONCLUSION}

Our results suggest that improving GP confidence in assessing and managing alcohol issues is key to facilitating routine alcohol enquiry. Although barriers such as competing health issues will inevitably influence GPs' priorities at consultation, building their clinical skills and confidence to identify, assess and manage problematic alcohol consumption could facilitate the integration of alcoholrelated discussions into routine care. Routine use of brief alcohol risk assessment instruments, such as the AUDIT-C, might improve confidence in assessing alcohol issues and reduce the time burden in risk assessment while de-stigmatising the nature of the 'morally charged nature of alcohol discussions'. This will ultimately reduce the potential for negative responses from patients.

Acknowledgements The authors gratefully acknowledge the contribution of 'HealthEd' seminar organisers, who assisted with recruitment, printed and administered the questionnaires, and entered the data.

Contributors ERM developed and implemented the survey, coordinated the statistical analysis and participated in the drafting of the manuscript. IJR participated in the drafting of the manuscript and LTT undertook all preliminary analyses and contributed to the drafting of the manuscript. GT contributed to the preliminary analysis and the drafting of the manuscript. RM participated in the survey design, administered and collated paper survey data and contributed to manuscript. GB and INO participated in the design of the study, commented on the analysis and helped draft the manuscript. All authors read and approved the final manuscript.

Funding This work was supported by the Australian Research Council, grant number LP 120200175, and Linkage Partners Cancer Council Australia and Drug and Alcohol Services of South Australia.

Competing interests None declared.

Ethics approval University of Adelaide Human Research Ethics Committee.

Provenance and peer review Not commissioned; externally peer reviewed.

Data sharing statement No additional data are available.

Open Access This is an Open Access article distributed in accordance with the Creative Commons Attribution Non Commercial (CC BY-NC 4.0) license, which permits others to distribute, remix, adapt, build upon this work noncommercially, and license their derivative works on different terms, provided the original work is properly cited and the use is non-commercial. See: http:// creativecommons.org/licenses/by-nc/4.0/

\section{REFERENCES}

1. Winstanley MH, Pratt IS, Chapman K, et al. Alcohol and cancer: a position statement from Cancer Council Australia. Med J Aust 2001;194:479-82.

2. Collins DJ, Lapsley HM. The costs of tobacco, alcohol and illicit drug abuse to Australian society in 2004/05: summary version. Canberra: Australian Government Department of Health and Ageing, 2008.

3. World Health Organization. WHO expert committee on problems related to alcohol consumption: second report. WHO Technical Report Series. Geneva: World Health Organization, 2007.

4. Britt $\mathrm{H}$, Miller GC, Henderson J, et al. General practice activity in Australia 2013-14. Sydney: Sydney University Press, 2014.

5. Teesson M, Baillie A, Lynskey M, et al. Substance use, dependence and treatment seeking in the United States and Australia: a cross-national comparison. Drug Alcohol Depend 2006;81:149-55.

6. Royal Australian College of General Practitioners. Guidelines for preventive activities in general practice, 9th edn. Melbourne: RACGP, 2016.

7. Tam CW, Zwar N, Markham R. Australian general practitioner perceptions of the detection and screening of at-risk drinking, and the role of the AUDIT-C: a qualitative study. BMC Fam Pract 2013;14:121.

8. Berner MM, Härter M, Kriston L, et al. Detection and management of alcohol use disorders in German primary care influenced by non-clinical factors. Alcohol Alcohol 2007;42:308-16.

9. Abouyanni G, Stevens LJ, Harris MF, et al. GP attitudes to managing drug- and alcohol-dependent patients: a reluctant role. Drug Alcohol Rev 2000;19:165-70. 
10. Grady A, Carey M, Oldmeadow C, et al. GP detection of health risk factors among general practice patients at risk of primary and secondary stroke. Fam Pract 2015;32:336-42.

11. Kaner EF, Beyer F, Dickinson HO, et al. Effectiveness of brief alcohol interventions in primary care populations. Cochrane Database Syst Rev 2007;2:CD004148.

12. Ballesteros J, Duffy JC, Querejeta I, et al. Efficacy of brief interventions for hazardous drinkers in primary care: systematic review and meta-analyses. Alcohol Clin Exp Res 2004;28:608-18.

13. Whitlock EP, Polen MR, Green CA, et al. Behavioral counseling interventions in primary care to reduce risky/harmful alcohol use by adults: a summary of the evidence for the U.S. Preventive Services Task Force. Ann Intern Med 2004;140:557-68.

14. Bertholet N, Daeppen J-B, Wietlisbach V, et al. Reduction of alcohol consumption by brief alcohol intervention in primary care: systematic review and meta-analysis. Arch Intern Med 2005;165: 986-95.

15. Wellard L, Corsini N, Hughes C. Discussing alcohol and cancer with patients: knowledge and practices of general practitioners in New South Wales and South Australia. Australian Fam Physician 2016;45:588-93.

16. Beich A, Gannik D, Malterud K. Screening and brief intervention for excessive alcohol use: qualitative interview study of the experiences of general practitioners. BMJ 2002;325:870.

17. Yoast RA, Wilford BB, Hayashi SW. Encouraging physicians to screen for and intervene in substance use disorders: obstacles and strategies for change. J Addict Dis 2008;27:77-97.

18. Anderson P, Wojnar M, Jakubczyk A, et al. Managing alcohol problems in General Practice in Europe: results from the European ODHIN survey of General Practitioners. Alcohol Alcohol 2016:51:630-630.

19. Knox SA, Harrison CM, Britt HC, et al. Estimating prevalence of common chronic morbidities in Australia. Medl $J$ Australia 2008;189:66-70.

20. Mules T, Taylor J, Price R, et al. Addressing patient alcohol use: a view from general practice. J Prim Health Care 2012;4:217-22.

21. Litt J. Exploration of the delivery of prevention in the general practice setting. Thesis. Adelaide: Flinders University, 2007.

22. Lock C, Wilson G, Kaner E, et al. A survey of general practitioners' knowledge, attitudes and practices regarding the prevention and management of alcohol-related problems: an update of a World Health Organisation survey ten years on. London: Alcohol Education and Research Council, 2009.

23. Mitchell AJ, Meader N, Bird V, et al. Clinical recognition and recording of alcohol disorders by clinicians in primary and secondary care: meta-analysis. British J Psychiatry 2012;201:93.

24. Harris MF, Hobbs C, Davies GP, et al. Implementation of a SNAP intervention in two divisions of general practice: a feasibility study. Med J Aust 2005;183:S54-8.

25. Denney-Wilson E, Fanaian M, Wan Q, et al. Lifestyle risk factors in general practice: routine assessment and management. Australian Fam Physician 2010;39:950-3.

26. Paul C, Yoong SL, Sanson-Fisher R, et al. Under the radar: a cross-sectional study of the challenge of identifying at-risk alcohol consumption in the general practice setting. BMC Fam Pract 2014; 15:74

27. Fucito UM, Gomes BS, Murnion B, et al. General practitioners' diagnostic skills and referral practices in managing patients with drug and alcohol-related health problems: implications for medical training and education programmes. Drug Alcohol Rev 2003;22:417-24.

28. Waldron A, McGrath M. Alcohol disorders and older people: a preliminary exploration of healthcare professionals' knowledge, in Ireland. Int J Therap Rehab 2012;19:352-8.

29. McAvoy BR, Donovan RJ, Jalleh G, et al. General Practitioners, Prevention and Alcohol-a powerful cocktail? Facilitators and inhibitors of practising preventive medicine in general and early intervention for alcohol in particular: a 12-nation key informant and general practitioner study. Drug Edu Prev Pol 2001;8:103-117.

30. Swan A, Sciacchitano L, Berends L. Alcohol and other drug brief interventions in primary care. Victoria: Turning Point Alcohol and Drug Centre, 2008.
31. Babor TF, Grant M, eds. Project on identification and management of alcohol-related problems. Report on phase II: a randomized clinical trial of brief interventions in primary health care. Geneva: World Health Organization, 1992.

32. Babor TF, Grant M, Acuda W, et al. Comments on the WHO report "brief interventions for alcohol problems": a summary and some international comments. Addiction 1994:89:657-78.

33. Heather N, ed. WHO collaborative project on identification and management of alcohol-related problems in primary health care: report on Phase IV. Geneva: World Health Organization, 2006.

34. Heather N. A long-standing World Health Organization collaborative project on early identification and brief alcohol intervention in primary health care comes to an end. Addiction 2007;102:679-81.

35. Babor TF, Higgins-Biddle JC. Brief intervention for hazardous and harmful drinking: a manual for use in primary health care. Geneva: The World Health Organization, 2001.

36. Fleming MF. Screening and brief intervention in primary care settings. Alcohol Res Health 2004-2005;28:57-62.

37. Fleming MF, Mundt MP, French MT, et al. Brief physician advice for problem drinkers: long-term efficacy and benefit-cost analysis. Alcohol Clin Exp Res 2002;26:36-43.

38. Kraemer KL. The cost-effectiveness and cost-benefit of screening and brief intervention for unhealthy alcohol use in medical settings. Subst Abuse 2007;28:67-77.

39. Mundt MP. Analyzing the costs and benefits of brief intervention. Health Serv Res 2006:29:34-6.

40. Wilk Al, Jensen NM, Havighurst TC. Meta-analysis of randomized control trials addressing brief interventions in heavy alcohol drinkers. J Gen Intern Med 1997;12:274-83.

41. Stockwell T. Chapter 6. Prevention. In: World Health Organization, ed. ATLAS on Substance use (2010): resources for the prevention and treatment of substance use disorders. edn. Geneva: World Health Organization, 2010:105-24.

42. Bush K, Kivlahan DR, McDonell MB, et al. for the Ambulatory Care Quality Improvement Project (ACQUIP). The AUDIT Alcohol Consumption Questions (AUDIT-C): an effective brief screening test for problem drinking. Arch Intern Med 1998:158:1789-95.

43. Aalto M, Alho H, Halme JT, et al. AUDIT and its abbreviated versions in detecting heavy and binge drinking in a general population survey. Drug Alcohol Depend 2009;103:25-9.

44. Kelly TM, Donovan JE, Chung T, et al. Brief screens for detecting alcohol use disorder among 18-20-year-old young adults in emergency departments: comparing AUDIT-C, CRAFFT, RAPS4-QF, FAST, RUFT-Cut, and DSM-IV 2-Item Scale. Addict Behav 2009;34:668-74.

45. Brady M, Sibthorpe B, Bailie R, et al. The feasibility and acceptability of introducing brief intervention for alcohol misuse in an urban Aboriginal medical service. Drug Alcohol Rev 2002;21:375-80.

46. Drummond C, Deluca $P$, Coulton $S$, et al. The effectiveness of alcohol screening and brief intervention in emergency departments: a multicentre pragmatic cluster randomized controlled trial. PLOS ONE 2014;9:e99463.

47. Yarnall KSH, Pollak KI, Østbye $\mathrm{T}$, et al. Primary care: is there enough time for prevention? Am J Public Health 2003;93:635-41.

48. Johnson M, Jackson R, Guillaume L, et al. Barriers and facilitators to implementing screening and brief intervention for alcohol misuse: a systematic review of qualitative evidence. J Public Health 2011;33:412-21.

49. Aalto M, Pekuri P, Seppä K. Primary health care professionals' activity in intervening in patients' alcohol drinking: a patient perspective. Drug Alcohol Depend 2002;66:39-43.

50. Miller PM, Thomas SE, Mallin R. Patient attitudes towards self-report and biomarker alcohol screening by primary care physicians. Alcohol Alcohol 2006:41:306-10.

51. Tam CW, Leong LH, Zwar N, et al. Consultation contexts and the acceptability of alcohol enquiry from general practitioners-a survey experiment. Australian Fam Physician 2015;44:490-6.

52. Tam CW, Leong L, Zwar N, et al. Alcohol enquiry by GPsUnderstanding patients' perspectives: a qualitative study. Australian Fam Physician 2015;44:833-8.

53. Sax LJ, Gilmartin SK, Bryant AN. Assessing response rates and nonresponse bias in web and paper surveys. Res High Educ 2003;44:409-32. 